\title{
GRUPOS OPERATIVOS DE APRENDIZAGEM NOS SERVIÇOS DE SAÚDE: SISTEMATIZAÇÃO DE FUNDAMENTOS E METODOLOGIAS
}

\author{
Operative Groups of Learning in the Health Services: \\ Basis and Methodologies Systematization \\ Los Grupos Operativos de Aprendizaj e en los Servicios de Salud: \\ Sistematización de Fundamentos y Metodologías
}

Sônia Maria Soares

Aidê Ferreira Ferraz

\begin{abstract}
Resumo
Trata-se de uma pesquisa com abordagem qualitativa, modalidade estudo de caso, que teve como objetivo analisar a aplicação da teoria e da metodologia dos grupos operativos, proposta por Pichon-Rivière em grupos de portadores de doenças crônico-degenerativas. Foi desenvolvida em um hospital universitário da cidade de Belo Horizonte - Minas Gerais/ Brasil, por meio de entrevistas e observação direta de um grupo de sete adultos com diabetes tipo 1, com idade entre 18 e 45 anos, durante os encontros no grupo operativo. A análise dos dados fundamentou-se no referencial dos indicadores do processo grupal, propostos por Pichon-Rivière (1998): afiliação e pertença, cooperação, comunicação, aprendizagem e pertinência. A aplicação da teoria e da metodologia proposta por Pichon-Rivière permitiu ao coordenador do grupo operativo e às pesquisadoras ampliar o olhar em relação à dinâmica grupal, o que contribuiu para que os conteúdos subjetivos, implícitos nas interações dos participantes fossem trabalhados, mas não verbalizados. Além disso, os indicadores do processo grupal permitiram reavaliar e adaptar o desenvolvimento do grupo operativo, norteando as ações desenvolvidas a cada encontro. Fundamentadas nos achados deste estudo, as autoras acreditam que esta pode ser uma estratégia eficaz para os profissionais de saúde, na construção de modelos que envolvam trabalhos com grupos.
\end{abstract}

Palavras-chave: Estrutura de Grupo. Serviços de Saúde. Diabetes Mellitus.

\begin{abstract}
It is a research with qualitative approach, case study modality, witch had as purpose to analyze the theoretical application and the methodology of the operative groups, proposed by Pichon-Rivière in groups of people carrier of chronic degenerative disease. The research was developed in a universitarian hospital of Belo Horizonte city - Minas Gerais/ Brazil, by interview and direct observation of a group of seven adults with diabetes type 1 , in age between 18 and 45 years old, during the meetings of the operative group. The analysis of the data was based in the indicatives of the group process referential, proposed by Pichon-Rivière (1998): affiliation and related, cooperation, communication, learning and importance. The application of the theory and of the methodology proposed by Pichon-Rivière allowed to the coordinator of the operative group and to the researchers to amplify the view about the group dynamics, what has contributed to the subjective contents, implicit in the participants' interaction was worked, but not verbalized. Besides this, the indicatives of the group process allowed to re-evaluate and to adapt the development of the operative group, guiding the developed actions in every encounter. Based in the founds of this study, the authors believe that this could be a, effectual strategy to the health professionals, in the construction of models that could involve group works.
\end{abstract}

\section{Keywords:}

Group Structure. Health Services. Diabetes Mellitus.

\section{Resumen}

Tratase de una investigación con abordaje cualitativa, modalidad estudio de caso, que tubo como objetivo analizar la aplicación de la teoría y de la metodología de los grupos operativos, propuesta por Pichon-Rivière en grupos de portadores de enfermedades crónico-degenerativas. Fue desarrollada en un hospital universitario de la ciudad de Belo Horizonte - Minas Gerais/Brasil, por medio de entrevistas y observación directa de un grupo de siete adultos con diabetes tipo 1, con edad entre 18 y 45 años, durante el encuentro en el grupo operativo. El análisis de los datos fue fundamentado en el referencial de los indicadores de proceso grupal, propuestos por Pichon-Rivière (1998): afiliación y pertenencia, cooperación, comunicación, aprendizaje y pertinencia. La aplicación de la teoría e de la metodología propuesta por Pichon-Rivière permitió al coordinador del grupo operativo y a las investigadoras ampliar el mirar en relación a la dinámica grupal, lo que contribuyó para que los contenidos subjetivos, implícitos en las interacciones de los participantes fuesen trabajados, pero non verbalizados. A más de los indicadores del proceso grupal permitieran reevaluar y adaptar el desarrollo del grupo operativo, norteando las acciones desarrolladas a cada encuentro. Fundamentadas en los hallados de este estudio, las autoras acreditan que esta puede ser una estrategia eficaz para los profesionales de salud, en la construcción de modelos que envuelvan trabajos con grupos.

Palabras clave:

Estructura de Grupo. Servicios de Salud. Diabetes Mellitus. 


\section{INTRODUÇÃO}

Uma longa tradição tem marcado a utilização de grupos na área da saúde. Na década de 1970, os grupos denominados operativos ganharam a atenção dos profissionais de saúde pelo seu grande potencial de aplicabilidade e pela sistematização que traziam para o processo grupal ${ }^{1,2}$.

A organização de grupos como modalidade de atenção coletiva à população tem sido cada vez mais freqüente nos serviços de saúde, o que se pode verificar pela participação de muitos clientes em vários grupos nos centros de saúde.

Em geral, esses grupos têm como critérios organizadores o tipo de doença, a idade e outros. Dentre os benefícios dessa modalidade de atendimento destacam-se: maior otimização do trabalho com a diminuição das consultas individuais, participação ativa do cliente no processo educativo e 0 envolvimento da equipe de profissionais com o cliente. Entretanto, percebe-se a falta de referências teóricas e metodológicas que orientem as ações dos profissionais na coordenação de grupos nos serviços de saúde.

É indispensável aos profissionais de saúde a discussão e a aprendizagem sobre os fenômenos grupais, com a finalidade de compreender os fundamentos teóricos da dinâmica grupal e ampliar o seu olhar sobre o grupo.

As doenças crônico-degenerativas vêm merecendo, por parte do setor saúde, atenção cada vez maior, seja pela sua elevada prevalência em nosso meio, como também pelo alto custo que representam para os serviços de saúde. 0 diabetes mellitus, por exemplo, é hoje um dos mais significantes problemas de saúde pública, tanto em número de pessoas afetadas quanto em número de incapacitações e mortalidade prematura. Segundo dados do Ministério da Saúde ${ }^{3}$, sabe-se que mais de $40 \%$ da clientela atendida na rede básica é prioritariamente composta de pessoas acometidas por doenças crônico-degenerativas.

É nesse contexto que os serviços de saúde têm enfatizado a importância do atendimento a essa clientela, seja no âmbito da atenção individual, seja no da coletiva, como é o caso dos grupos operativos. A partir desse quadro, o Ministério da Saúde, em parceria com as Secretarias Estaduais, Municipais e a Estratégia de Saúde da Família em todo território nacional, criou, em novembro de 2000, o Plano de Reorganização da Atenção à Hipertensão Arterial e ao Diabetes Mellitus ${ }^{3}$ com a finalidade de capacitar os profissionais de saúde que atuam na área da atenção básica, destacando-se nessa proposta a especificidade do trabalho com grupos operativos e a sua importância no acompanhamento e no controle do cliente.

Diante do exposto, este estudo poderá ser um elemento facilitador para a construção e a sistematização de metodologia visando à sua aplicação mais definida para a educação permanente de profissionais e a formação do estudante na área da saúde. Assim, evidencia-se a necessidade de sistematização de metodologias com a finalidade de capacitar os profissionais de saúde para esse tipo de intervenção.

\section{OBJ ETIVO}

Analisar a aplicação da teoria e da metodologia dos grupos operativos, proposta por Pichon-Rivière em grupos de portadores de diabetes mellitus e sua contribuição à assistência e ao ensino na área da saúde.

\section{REFERENCIAL TEÓRICO}

Foi Pichon-Rivière ${ }^{4}$, psiquiatra e psicanalista argentino, quem elaborou, na década de 1940, a teoria do grupo operativo. Ele definiu o grupo como um conjunto de pessoas, ligadas no tempo e espaço, articuladas por sua mútua representação interna, que se propunham, explícita ou implicitamente, a uma tarefa, interatuando em uma rede de papéis com o estabelecimento de vínculos entre si. Assim, a teoria dá grande importância aos vínculos sociais, que são a base para os processos de comunicação e aprendizagem, considerando que o ser humano é essencialmente um sujeito social. 0 grupo se põe como uma rede de relações com base em vínculos entre cada componente e todo o grupo e vínculos interpessoais entre os participantes ${ }^{4}$.

0 grupo se une em torno de uma tarefa, que é compreendida em nível consciente, mas que também implica uma dimensão afetiva que existe no inconsciente do grupo. Assim, um nível de funcionamento do grupo é lógico e relacionado à tarefa e outro está envolvido com as emoções e a dinâmica psíquica do grupo, seus medos e fantasias. Os objetivos conscientes do grupo delimitam a sua tarefa externa. Mas ele também tem uma tarefa interna, que é trabalhar com os processos vividos, em nível consciente e inconsciente, que dificultam ou mesmo impedem a realização da tarefa externa ${ }^{4,5}$.

Conforme nos explicam Portarrieu e Tubert-0aklander ${ }^{6}$, 0 grupo operativo constitui uma modalidade de processo grupal que, em princípio, deve ser: dinâmico - permitindo-se o fluir da interação e da comunicação para fomentar o pensamento e a criatividade; reflexivo - uma parte da tarefa é a reflexão sobre o próprio processo grupal, particularmente quando se trata de compreender os fatores que obstruem a tarefa; e democrático quanto à tarefa - 0 grupo origina suas próprias ações e pensamentos, em um princípio de autonomia.

Todo grupo, ao formular seus objetivos, se propõe a uma mudança ou realização de uma tarefa; mas também apresenta um grau menor ou maior de resistência a essa mudança, diante da qual evidenciam-se os medos básicos de perda e de ataque; isto é, de um lado, o medo de perder o que já se tem - até mesmo a própria identidade - e que se relaciona a uma ansiedade depressiva, e, de outro, o medo do desconhecido, que se liga a uma ansiedade paranóica ou persecutória. Tal resistência à mudança provoca entraves psíquicos e afetivos à aprendizagem e à comunicação no grupo. Inspirado no filósofo Gaston Bachelard, Pichon-Rivière denomina esses entraves de obstáculos epistemofílicos ${ }^{4,5,6,7}$. Obstáculos que representam as dificuldades de apreender um objeto de conhecimento para efetuar uma leitura correta da realidade. 
Os processos vividos no grupo geram uma ressonância, ou seja, o processo individual entra no campo grupal provocando identificações e reações em cadeia, conforme a distância psíquica entre os membros. Assim, um membro serve de suporte para processos psíquicos de outros membros e do grup $0^{4,5,6}$.

Conseqüentemente, o trabalho com o grupo visa à integração de duas dimensões: a verticalidade, que se refere à história de cada participante, e que o leva a uma reatualização emocional no grupo e a um processo transferencial; e a horizontalidade, que se refere ao campo grupal, consciente e inconsciente, que vai sendo modificado pela ação e interação dos membros ${ }^{4,5}$.

No grupo operativo, instrumentaliza-se um processo que passa fundamentalmente pela diminuição dos medos básicos, com o fortalecimento do Eu e uma adaptação ativa à realidade. Hierarquiza-se, como tarefa grupal, a construção de um Esquema Conceitual, Referencial e Operativo (ECRO) comum, condição necessária para a comunicação e a realização da tarefa. A tarefa depende, portanto, do campo operativo do grupo: sua percepção, sua interação, sua linguagem ${ }^{4,5,6}$.

Mas, se a realização da tarefa acrescenta experiência ao grupo, ela também o força a rever seus conflitos e formas de organização, de forma que o processo grupal envolve uma constante desestruturação e reestruturação desse campo e dos sujeitos nele envolvidos ${ }^{4}$.

No processo do grupo, há os momentos da pré-tarefa, da tarefa e do projeto. A pré-tarefa é o momento em que predominam mecanismos de dissociação, com a finalidade de defender os sentimentos de culpa e ambivalência, a situação depressiva básica, as dificuldades de tolerância, a frustração e a postergação. A tarefa é o momento em que se rompe a estereotipia e se elabora a pré-tarefa, avançando na elaboração de seu objetivo. Nesse momento, alcança-se maior operatividade e criatividade, podendo-se sistematizar objetivos e realizar tarefas propostas e/ou novas. No momento do projeto, uma vez alcançado um nível de operatividade, 0 grupo pode se planejar $3,4,5,6$.

0 grupo vai se tornando mais consciente e flexível quanto aos seus papéis, centrando-se no rompimento de estereótipos e na modificação de vínculos internos e externos. Centra-se no campo grupal. Cada indivíduo, ao expressar-se, é portavoz de uma dimensão ou especificidade do campo grupal ${ }^{7,8}$.

Esses momentos do grupo não seguem uma lógica linear e cumulativa. Pelo contrário, todo grupo apresenta ambivalências, regressão, dispersão diante da constante demanda de sustentar seu processo e refletir sobre ele. Isso significa que ele tem de estar continuamente se reorganizando e se recriando. Ou seja, é necessário que se proceda a revisão em um sentido imaginário, a destruição - de seus ideais para que se possa reconstruí-los no contexto, procurando a realização da tarefa. Pichon-Rivière ${ }^{4}$ aponta então para uma recriação do objeto destruído, isto é, a recuperação de uma imagem do grupo e dos seus objetivos, mas sempre de forma renovada ${ }^{4,7}$.

Pichon-Rivière apresenta a concepção de uma espiral dialética relativa aos momentos do grupo, ou seja, diante da situação grupal, uma interpretação é gerada e provoca uma desestruturação, e, a seguir, o grupo responde tentando se transformar para dar conta de seu processo, passando por uma reestruturação, em uma nova situação ("emergente"). Cada ciclo abrange e supera 0 anterior $4,6,8,910$.

A espiral dialética ${ }^{4}$ abrange 0 todo do processo grupal, como um movimento constante entre processos internos ao grupo. Esse movimento pode ser observado tomando como referência os indicadores do processo grupal, quais sejam: afiliação/pertença, comunicação, cooperação, tele, aprendizagem e pertinência.

A afiliação e a pertença dizem respeito ao grau de identificação dos membros do grupo entre si e com a tarefa. Enquanto a afiliação indica apenas uma aquiescência em pertencer ao grupo, a pertença envolve um sentimento de identificação com o grupo. A pertença possibilita a identidade, mas também contém a diferenciação. A afiliação e a pertença são indicadores básicos para o desenvolvimento dos outros processos no grupo.

A cooperação pressupõe ajuda mútua e se dá mediante 0 desempenho de diferentes papéis e funções. Na tentativa de articular demandas do grupo e dos indivíduos, muitas vezes surge a competição. A flexibilização dos papéis é uma forma de se trabalhar esse problema, buscando a "co-operatividade". "Co-operar" não significa não discordar ou confrontar, mas, sim, atuar quando se é cúmplice (ou não ser cúmplice daquilo de que se discorda). Cooperação e comunicação interligam-se e favorecem a aprendizagem.

A comunicação é um processo que leva em conta as redes de comunicação no grupo, contendo possibilidades e entraves. Envolve também o conflito e a necessidade de trabalhar sobre ele. É preciso elaborar o que se chama de "mal-entendido", que está associado a conflitos diversos, tanto aqueles relacionados à organização do grupo quanto os concernentes a conflitos psíquicos ${ }^{789}$. A aprendizagem vai além da mera incorporação de informações e pressupõe o desenvolvimento da capacidade de criar alternativas - por meio dela percebe-se o grau de plasticidade grupal diante dos obstáculos e da criatividade para superar as contradições e mesmo integrá-las. Com a atenuação da ansiedade básica, o grupo pode operar melhor seus afetos e sua tarefa. A aprendizagem está inter-relacionada à comunicação, e o grupo precisa compreender seus obstáculos à comunicação para analisar os obstáculos à aprendizagem. Ao mesmo tempo, é apenas na dimensão da tele que o grupo consegue deslanchar todos os seus outros processos.

A tele caracteriza a disposição positiva ou negativa dos membros do grupo entre si. Refere-se às relações no grupo tais como são percebidas e vividas. É uma disposição para atuar em conjunto e, assim, pode ser positiva ou negativa. As percepções entre os membros do grupo estão vinculadas aos processos transferenciais. Assim, a tele aparece como uma rede de transferências.

É importante assinalar que, para Pichon-Rivière ${ }^{4}$, o que se encontra no grupo não é uma neurose transferencial, mas, sim, processos transferenciais em uma rede de relações. A transferência é um processo de atribuição de papéis ao outro com base nas expectativas inscritas na vida psíquica do sujeito. 
Comporta a reprodução de sentimentos inconscientes, que indica a reprodução estereotipada de situações, característica de uma forma de adaptação passiva, na qual o sujeito se vê atado a conflitos psíquicos não trabalhados. Essa reprodução tem o efeito de proteger contra o medo da mudança e, portanto, de fortalecer a resistência a ela ${ }^{4,7}$.

A pertinência refere-se à produtividade do grupo, à sua capacidade de centrar-se em seus objetivos, de forma coerente com seus outros processos. A realização de objetivos em um contexto requer pertinência do agir, que se afasta tanto do conformismo quanto da ruptura total do contexto.

Esses processos do grupo não são estanques e nem lineares. Há um constante ir-e-vir entre os momentos. Para PichonRivière $^{4}$, são aspectos do processo grupal que interatuam de forma dinâmica permitindo mudanças no seu desenvolvimento.

\section{METODOLOGIA}

Trata-se de uma pesquisa na abordagem qualitativa na modalidade de estudo de caso. 0 estudo foi desenvolvido no serviço de endocrinologia de um hospital universitário de Belo Horizonte - MG/Brasil.

A coleta de dados constou de entrevista durante a consulta de enfermagem e observação de um grupo de sete adultos, sendo cinco mulheres e dois homens com diabetes mellitus tipo 1 , na faixa etária entre 18 e 45 anos, no período de novembro de 2003 a julho de 2004. Os princípios ético-legais foram observados conforme previsto na Resolução 196/96 do Conselho Nacional de Saúde. As atividades grupais foram desenvolvidas em encontros quinzenais, compreendendo as seguintes etapas: identificação dos clientes diabéticos para inserção no grupo; planejamento das atividades do grupo; observação e descrição do processo grupal; e elaboração e reflexão desse processo.

Inicialmente, cada cliente foi submetido a uma avaliação individual por meio da consulta de enfermagem, onde foram levantados os principais fatores de risco para o aparecimento de complicações agudas e crônicas, além de ser avaliado o seu potencial para o autocuidado. As etapas subseqüentes constaram de atividades grupais, nas quais foram discutidas crenças em relação ao diabetes, os sentimentos que emergiram após o diagnóstico, temas relativos ao autocuidado para o controle da doença, riscos de complicações agudas e crônicas.

Os participantes foram submetidos a uma segunda consulta de enfermagem três meses após o início do trabalho de campo, com a finalidade de avaliar o controle da doença e o impacto imediato da sua inserção no grupo. Nessa etapa foram utilizados jogos educativos para avaliar os indicadores relacionados ao processo grupal e o seu potencial para o autocuidado.

Os grupos foram conduzidos pela pesquisadora e uma aluna do curso de graduação de Enfermagem, que atuou como observadora do grupo operativo, registrando cada encontro e elaborando relatórios de campo. A observação foi feita mediante o registro cursivo das reuniões do grupo, considerando os indicadores do processo grupal propostos por Pichon- Rivière 4 .

\section{ANÁLISE E DISCUSSÃO DOS RESULTADOS}

\section{Caracterização do grupo}

Cinco participantes do grupo dedicavam-se ao trabalho doméstico, um era aposentado por invalidez e outro trabalhava como cobrador de ônibus. 0 nível de escolaridade de seis participantes é o ensino fundamental incompleto, apenas uma participante tinha o curso superior incompleto. 0 tempo de diagnóstico da doença foi bem diversificado, sendo que três pessoas tinham mais de cinco anos de convivência com a doença, enquanto os demais tinham menos de dois anos.

\section{Demanda e formação dos grupos}

Observou-se que o critério organizador do grupo apoiavase nos seguintes aspectos: o diagnóstico de diabetes mellitus, a idade, o processo de tratamento, a avaliação dos resultados de exames e o tempo de convivência com a doença.

Desde o primeiro encontro foi formalizado o contrato com o grupo, no qual foram definidos como e quando seriam realizadas as reuniões, os horários, os temas que seriam trabalhados, as expectativas dos participantes e a apresentação do coordenador.

A forma de organização do grupo confirmava que a patogênese era o eixo norteador para abordagem clínica do mesmo com enfoque no modelo biomédico e na doença ${ }^{11,12}$.

\section{Desenvolvimento do grupo}

0 desenvolvimento do grupo ocorreu nas seguintes etapas: primeiro, cada participante foi avaliado individualmente para a realização do exame de glicemia capilar, controle de peso e sinais vitais. Em seguida, foi discutido um tema escolhido anteriormente pelo grupo com o apoio de jogos educativos e dinâmicas.

A realização do exame de glicemia capilar para alguns pacientes, mesmo convivendo com a doença por muitos anos, ainda despertava resistência e tensão, como menciona uma das participantes:

Eu não me acostumei com essa coisa de furar o dedo... não consigo nem olhar...

A abordagem dos temas de interesse do grupo ocorreu por meio de dinâmicas e vivências ${ }^{11}$, que incentivavam as pessoas a falarem sobre si, daquilo que gostavam de fazer no seu cotidiano de convívio com a doença, e a linha da vida que discutiu as mudanças ocorridas antes e depois da doença por meio de desenhos que expressavam as dificuldades de seu controle, como menciona uma das participantes:

Eu curto e faço esporte duas vezes por semana. O que eu não curto e faço é fumar... Eu não curto e não faço é beber, não gosto. Eu curto e não faço é ir em festas, eu adoro e não faço muito, gostaria de fazer mais.

Esse foi um momento lúdico do grupo, que permitiu a cada membro se apresentar e falar de fatos que constituem a sua vida cotidiana, o seu trabalho, a sua família, a sua vida social. 0 fato de o grupo operativo falar do cotidiano é importante como forma de cada um ir reconhecendo o outro, suas necessidades e aquilo que identifica cada um²,10. 


\section{Temas emergentes}

Emergiram da discussão nos grupos as seguintes unidades temáticas: a revolta e o inconformismo no enfrentamento da doença; a consciência sobre os riscos de complicações advindas da doença; o inconformismo com a falta de assistência de saúde; a dificuldade em aderir à dieta; a influência emocional no controle do diabetes; e o medo das complicações futuras. Os depoimentos abaixo expressam cada um dos temas emergentes:

... 17 anos descobri o diabetes, foi uma revolta, estava na adolescência, foi difícil demais. Acho que o problema é a comida, a gente não pode comer e fica com vontade. [relato do participante se referindo a dificuldades de freqüentar festas.]

É muito melhor fazer bem a dieta do que ficar uma semana no hospital, eu te garanto. Perdi o braço por causa de um acidente, o pronto-socorro engessou apertado, o sangue não circulou, a diabetes alterou e não conseguiram controlar, tiveram que amputar...

Na finalização de cada encontro, era reafirmado o contrato grupal e eram discutidas as datas dos próximos encontros, horários e temas que seriam trabalhados.

\section{Elaboração de material didático}

Para o desenvolvimento das atividades foram elaborados folderes explicativos sobre complicações agudas e crônicas do diabetes mellitus e jogos educativos que discutiam temas sobre o uso de medicamentos orais, dieta e exercício físico. 0 jogo sobre dieta foi um dos mais utilizados. Ele propiciava ao cliente combinação de cardápios diferenciados, que eram discutidos em grupo, avaliando a composição e a combinação alimentar sobre o que devem e o que não devem comer e como preparar seus alimentos.

\section{Os indicadores do processo grupal}

Retomando os indicadores de avaliação e acompanhamento do processo grupal propostos por Pichon- Rivière ${ }^{4}$, observa-se que, em relação à afiliação e à pertença, desde o primeiro encontro os integrantes demonstraram o interesse em estar no grupo, expresso no compromisso com a participação em todas as atividades propostas, indicadores que foram aumentando gradativamente a cada reunião.

Mesmo com o interesse em discutir temas do cotidiano que emergiam no grupo, como o desemprego, a violência nos grandes centros urbanos, observou-se que foi preponderante na pertinência do grupo a tarefa de aprender o manejo do diabetes. Com objetivos comuns, os membros do grupo trocaram conhecimentos sobre 0 diabetes, além de exporem suas experiências e dificuldades, aprendendo, assim, a conviver com a doença.

A cooperação foi demonstrada à medida que os membros do grupo buscaram complementar o outro com base em situações que eram trazidas do seu cotidiano. Isso ficou mais evidente quando um dos membros do grupo teve a confirmação que precisaria fazer uma pequena cirurgia, mas a glicemia estava muito descontrolada. Todos demonstraram solidariedade, e duas se ofereceram como acompanhantes.
A comunicação no grupo não ocorreu de forma linear, ou seja, apenas entre coordenador e participante. De modo geral, os participantes processavam uma comunicação integrada, entre eles próprios e entre a coordenação.

A aprendizagem foi claramente observada nos participantes do grupo. Novos conhecimentos foram adquiridos, o que foi explicitado pela preocupação com o manejo da doença e melhoria do controle glicêmico. Os integrantes que já tinham maior convivência com a doença expressaram preocupação com a qualidade de vida. A oportunidade de rever como estava sendo realizado o tratamento e o seu acompanhamento, nem sempre bem-sucedido, trouxe reflexões sobre o estilo de vida e o resgate de matrizes de aprendizagem que foram marcantes para as mudanças que cada um sabe que precisa realizar no seu cotidiano de vida. A relação dos participantes ocorreu de forma harmoniosa e caracterizou-se pelo respeito às diferenças.

No tocante aos momentos do processo grupal, observou-se que nos primeiros encontros os participantes se mostraram receosos, possivelmente porque ainda não se conheciam. As primeiras impressões foram se formando e, mesmo participando dos momentos iniciais do trabalho em grupo, essa participação não era integral. Em geral, os clientes não se identificavam com as atividades do grupo, mostrando-se na fase de pré-tarefa. Depois de alguns encontros, os integrantes foram se desinibindo e se integrando ao grupo, contando suas experiências, propondo atividades. A partir de então, todos já se conheciam e as impressões do primeiro encontro foram dando lugar ao desejo de interagir e de atingir os objetivos que se tornaram comuns. Nessa etapa, o grupo já estava mais integrado com a tarefa.

Em relação à tele, observamos que houve uma disposição positiva dos membros do grupo entre si, o que facilitou a atuação do coordenador e a interação entre os membros do grupo.

\section{Os processos intragrupos}

Um dos participantes do grupo, ao assumir uma posição de resistência, mantinha-se em pré-tarefa, sabotando as atividades que estavam previstas para o desenvolvimento do mesmo.

Observou-se no grupo que tanto os aspectos da horizontalidade como os da verticalidade, que integram os processos intragrupos, mobilizaram ações capazes de modificar conceitos, crenças e valores em relação à doença.

\section{O papel do coordenador no processo grupal}

No grupo operativo, a principal função do coordenador, após estabelecer um enfoque adequado para a operação do grupo, é ajudar, por meio de intervenções interpretativas, o grupo a realizar sua tarefa interna reflexiva, para colocar-se em condições de desenvolver sua tarefa externa. A explicitação e a interpretação dos fatores implícitos no acontecer grupal permitem aos membros tomar consciência e enfrentar obstáculos que, ao permanecerem inconscientes, continuariam a interferir na realização da tarefa externa ${ }^{12}$.

Nos grupos atendidos na área da saúde, pode-se pensar até que ponto o processo grupal é específico, por exemplo, em relação à demanda por saúde, às fantasias e aos medos relativos à doença que identifica a escolha de participantes. 
Assim, o coordenador poderá conduzir melhor o grupo se puder interligar as dificuldades diante da tarefa externa (a necessidade de mudar os hábitos alimentares dos componentes do grupo) e ansiedades e angústias que essa tarefa provoca, como o medo de ser estigmatizado pela família ou pelo círculo de amigos e que pode ser vivida por um ou mais participantes do grupo. Assim, a troca de experiências entre eles pode ser esclarecedora e motivadora para a mudança necessária.

0 coordenador realizou seu papel assimétrico assinalando diferenças, esclarecendo dúvidas, estabelecendo um contrato com o grupo, que foi sempre respeitado. Ressalte-se que as intervenções realizadas pelo coordenador foram apropriadas, ajudando o grupo no processo de contenção e interação.

\section{CONSIDERAÇÕES FINAIS}

A teoria de grupos operativos proposta por Pichon-Rivière ${ }^{4}$ permitiu organizar o fazer grupal com referências conceituais e sistematizadas, o que facilitou de forma significativa a coordenação do grupo. Além disso, propiciou aos clientes um espaço terapêutico de discussão, no qual foi possível confrontar as fantasias inconscientes e medos sobre a repercussão do diabetes no seu cotidiano, contribuindo para o seu crescimento pessoal.

Observou-se que a experiência do grupo trouxe aprendizagem para melhor convivência com a doença, possibilitando a alguns dos participantes a ressignificação da experiência, o que transcendeu as limitações que ela provoca. Todo grupo tem a possibilidade de em algum momento se tornar um grupo de processo que contribua para que tudo 0 que emerge na sua dinâmica seja expresso e interpretado de forma contextualizada e no nível real. Assim, observou-se que os indicadores do processo grupal permitiram interpretar e reavaliar o desenvolvimento do grupo operativo, norteando as ações desenvolvidas a cada encontro. Certamente que essa pode ser uma estratégia, para os profissionais de saúde, na construção de modelos que envolvam os trabalhos com grupos.

\section{Referências}

1. Fernandes MT. 0 trabalho com grupos na Saúde da Família: concepções e estratégias para o cuidado transcultural. [dissertação de mestrado]. Belo Horizonte (MG): Escola de Enfermagem, Universidade Federal de Minas Gerais; 2006

2. Soares SM. Os grupos operativos de aprendizagem nos serviços de saúde:uma análise crítica segundo a concepção de Pichon-Rivière. Anais do $48^{\circ}$ Congresso Brasileiro de Enfermagem; 1996 out. 06-14; São Paulo(SP), Brasil.1996. São Paulo(SP): Aben; 1997.

3. Ministério da Saúde(BR). Manual de hipertensão arterial e diabetes mellitus: plano de reorganização da atenção à hipertensão arterial e ao diabetes mellitus. Brasília(DF); 2002.

4. Pichon-Rivière E. O processo grupal. São Paulo(SP): Martins Fontes; 1998.

5. Baremblitt G. Grupos: teoria e técnica. Rio de Janeiro(RJ): Graal; 1996.

6. Portarrieu ML, Tubert-Oklander J. Grupos operativos. In: Osório LC, organizador. Grupoterapia hoje. Porto Alegre(RS): Artes Médicas; 1989.

7. Berstein M. Contribuições de Pichón-Rivière à psicoterapia de grupo. In: Osório LC, organizador. Grupoterapia hoje. Porto Alegre(RS): Artes Médicas; 1986.

8. Osório LC, organizador. Grupoterapia hoje. Porto Alegre(MG): Artes Médicas; 1986.

9. Osório LC. Grupos, teorias e práticas: acessando a era da grupalidade. Porto Alegre(RS): Artes Médicas, 2000

10. Gayotto MLC, Domingues I. Liderança: aprenda a mudar em grupo. Petrópolis(RJ): Vozes, 1998

11. Minicucci A. Dinâmica de grupo: teorias e sistemas. $4^{\mathrm{a}}$ ed. São Paulo(SP): Atlas; 1997.

12. Andaló C. 0 papel de coordenador de grupo. Psicol. USP 2001;12(1): 135-52.

\section{Sobre as Autoras}

\section{Sônia Maria Soares}

Enfermeira. Doutora em Saúde Pública, Profa. Adjunta da Escola de Enfermagem da UFMG - Departamento de Enfermagem Básica. Coordenadora do Núcleo de Estudos e Pesquisas em Cuidado e Desenvolvimento Humano.

E-mail: smsoares.bhz@terra.com.br.

\section{Aidê Ferreira Ferraz}

Enfermeira. Doutora em Enfermagem, Profa. Associada da Escola de Enfermagem da UFMG - Departamento de Enfermagem Básica. Coordenadora do Núcleo de Estudos e Pesquisas sobre Comunicação em Enfermagem - NEPCE. E-mail: afferraz@terra.com.br. 